\title{
Prevention of coronary restenosis by stenting
}

\author{
U. Sigwart, U. Kaufmann, J.-J. Goy, M. Grbic, S. Golf, A. Essinger, A. Fischer, H. Sadeghi, \\ V. MIRKOVITCH AND L. KAPPENBERGER \\ Centre Hospitalier Universitaire Vaudois, CH-1011 Lausanne, Switzerland
}

KEY WORDS: Restenosis, coronary angioplasty, vascular stents.

Balloon angioplasty fails to provide acceptable long-term results for a significant proportion of patients. An intravascular mechanical support, developed with the aim of preventing restenosis and acute closure of diseased arteries after transluminal angioplasty, was implanted in 44 patients ( 39 male and five female), aged from 35 to 70 years (mean 56 years) with documented restenosis of native coronary artery (4l stents) and bypass grafts (12 stents). In the group of bypass graft patients there was no local restenosis and no major complication. In patients in whom stents were placed in native coronary arteries, the complication rate was higher ( $t$ wo patients died after coronary bypass surgery). One patient died suddenly at home. Except for one patient, in whom a new lesion developed proximally with extension into the stent, no case of restenosis could be observed.

Despite the still relatively high complication rate, we feel that stenting may present a rational approach to the unresolved problem of restenosis after coronary angioplasty.

\section{Introduction}

Restenosis after transluminal coronary angioplasty (PTCA) remains an unresolved problem. About one third ${ }^{[1]}$ of all patients will return within the first three months after PTCA for a second or third procedure thus reducing the benefit of an otherwise elegant and comparatively inexpensive procedure.

Despite significant improvements in coronary angioplasty equipment, the basic mechanism of PTCA has not changed in the 10 years of its existence. Therefore, it is not surprising that the rate of restenosis has remained relatively constant over many years. Modifications of procedural factors, such as balloon size, inflation presence, and number and duration of inflations, have failed to enhance the long-term results; in addition, drugs like calcium antagonists, platelets inhibitors, steroids and anticoagulants have not proved to induce restenosis rate $^{[2]}$. We have therefore investigated whether a mechanical intraluminal support implanted immediately after PTCA may provide a solution to the problem of restenosis.

\section{Methods}

A self-expanding, tubular stent prosthesis consisting of a woven stainless-steel mesh (Fig. 1) was developed in collaboration with Medinvent SA,
Lausanne. The stent can be constrained on a angioplasty-like delivery catheter the outer diameter of which does not exceed $1.5 \mathrm{~mm}$. The delivery catheter is advanced over a long exchange wire which remains in situ after the preceding angioplasty, and the retaining doubled-over membrane is then progressively withdrawn, thus deploying the stent in its unconstrained state at the site of the dilated lesion ${ }^{[3]}$.

\section{Patient selection}

Patients were enrolled in the study between April and July 1986; there were 39 male and 5 female patients, with ages ranging from 35 to 70 years (mean 56 years). All patients showed restenosis after previous PTCA; most patients had undergone two or three dilatations of the offending lesion before stent implantation and the interval between previous PTCA and re-dilatation with stenting averaged 9 weeks. All patients had objective and subjective signs of myocardial ischaemia according to history and a stress electrocardiogram. Informed consent (as per Helsinki declaration) was obtained from each patient and the study was approved by the Hospital Ethics Committee.

\section{Study protocol}

In view of the potential problems and hazards associated with intracoronary stents we preferred 

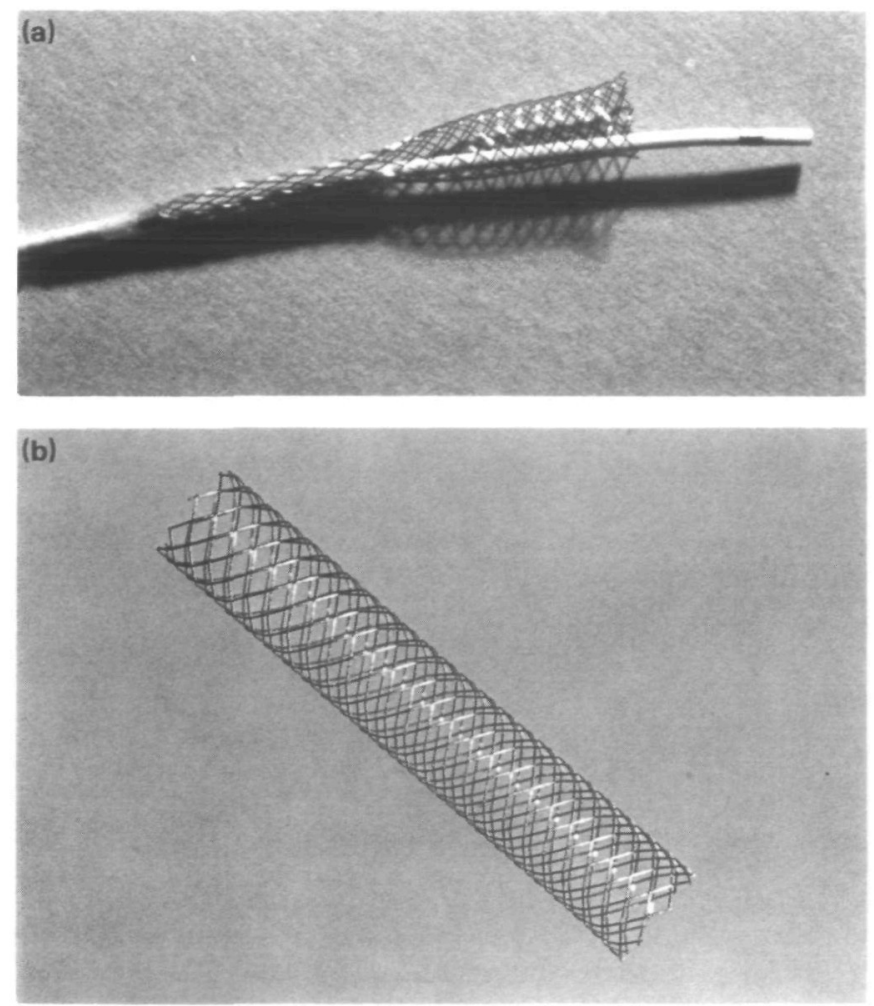

Figure I The stainless steel, multi-filament, self-expanding, macroporous prosthesis during use: (a) mounted on the delivery catheter; (b) fully expanded in the unconstrained state.

to consider our initial experience as an open study and, therefore, a control group was not used. The indication for stent implantation was posed by two cardiologists and one cardiovascular surgeon. The following were considered to be contraindications: lesions longer than $20 \mathrm{~mm}$, stenoses immediately adjacent to the left main bifurcation or to the origin of a large diagonal or marginal branch, impossibility of adequate follow-up, and intolerance to the concomitant drug treatment.

Patients were given $1 \mathrm{~g}$ of aspirin on the day prior to the procedure. One hour before cardiac catheterization they received $10 \mathrm{mg}$ of diazepam p.o. together with $10 \mathrm{mg}$ of nifedipine p.o. Before balloon dilatation, 10000-15000 units of heparin were injected through the arterial catheter; the last 15 patients also received $0.2 \mathrm{mg}$ intracoronary nifedipine. Heparin ( 5000 units) was added if the procedure lasted more than $90 \mathrm{~min}$. Balloon angioplasty was carried out using standard equipment. After the last balloon inflation, a $300 \mathrm{~cm}$ long wire
$(0.014$ in) was inserted and flushed with small amounts of urokinase during the exchange process. The stent delivery catheter was advanced over the exchange guide wire until it had passed the freshly dilated lesion. The correct position of the constrained stent was assured by multiple injections of contrast before and during the deployment in order to allow for last-minute adjustment. In three cases the lesion was not ideally covered by one single stent: a second stent was implanted proximally overlapping the first by $1-2 \mathrm{~mm}$.

Intracoronary nitrates were only administered if there was evidence of spasm following the stent deployment. After the procedure, the patients were observed in the intensive care unit and a heparin drip was started; partial thromboplastin time (PTT) was maintained for $70-90 \mathrm{~s}$. All patients received oral anticoagulants (acenocoumarin) for three months in addition to their antiplatelet regimen; the latter consisted of aspirin $(330 \mathrm{mg}$ ) plus dipyridamol $(75 \mathrm{mg})$ three times per day or, more recently, 
aspirin (100 mg) plus dipyridamol $(400 \mathrm{mg})$ and sulfinpyrazon $(400 \mathrm{mg}$ ) in two divided doses per day following a suggestion by Fuster ${ }^{[4]}$. Prevention of vasospasm was also pursued for three months by adding $20 \mathrm{mg}$ of nifedipine three times per day. The patients were discharged from the hospital once oral anticoagulation was stable, and were closely followed by frequent examination. Stress tests were performed after six weeks and three months, and control coronary angiography was scheduled 3-6 months after implantation; four patients had a second control coronary angiogram after one year.

\section{Results}

Among all patients who received intracoronary stents, 44 were treated for restenosis. A total of 53 stents were implanted in 49 sessions. Of these, 24 stents were implanted in the left anterior descending coronary artery (LAD), one of which was implanted in the first diagonal branch. Only one patient in this group had a double stent placed in the proximal LAD. Thirteen stents were implanted in the right coronary artery including two consecutive stents in the same vessel. Four single stents were placed in the circumflex coronary artery. Twelve stents were implanted in bypass grafts; two patients had three stents placed in the same bypass graft (one in two sessions and one in three sessions) and one patient had two stents placed in different bypass grafts during the same session.

The first follow-up angiogram $24 \mathrm{~h}$ after the procedure showed patency in all but one patient. In this particular patient the offending vessel was a left anterior coronary artery which perfused a partially infarcted region. There was evidence of collateral flow, but, because the patient was symptomatic with angina class II, it was decided to dilate the proximal $90 \%$ lesion which had restenosed after previous angioplasty. The stent was placed without difficulties; the immediate post-operative angiogram, however, revealed evidence of thrombotic material within the stent lumen. At that time, it was not customary to redilate the stent after placement with the angioplasty balloon. The stent was found to be occluded at the time of coronary angiography the next morning. It was decided not to recanalize the occlusion.

One early occlusion occurred three days after a placement following a maximal stress test. The stent was recanalized and remained open for some time but was found to be reclosed at the four-months follow-up. In addition, one patient had a suspected occlusion (also after maximal stress test) three days after implantation; this patient was operated on for an internal mammary artery bypass graft. At the time of operation no evidence of a thrombosis could be found. The case of this patient has been reported elsewhere $^{[3]}$. One patient with a long lesion $(23 \mathrm{~mm})$ of the proximal left anterior descending coronary artery, in the presence of a highly stenosed bypass graft to the same artery, underwent angioplasty and stenting with excellent immediate result. The stent was placed with its proximal end very close to the bifurcation to the circumflex artery without, however, interfering with it. The patient was asymptomatic for almost three months; he then developed angina again and was found to have a high-grade stenosis of the left main coronary artery extending into the stent. It was decided to submit this patient to his third elective bypass operation from which he did not recover, due to surgical complications. One patient with restenosis at the junction of a vein graft to the left anterior descending coronary artery (LAD) had a myocardial infarct due to occlusion of the distal vessel three months after the stent implantation. One patient with two stents in the proximal left anterior descending coronary artery died suddenly at home; it turned out that he had refused to take the prescribed medication after hospital discharge. Unfortunately no autopsy could be performed.

All other patients had an uneventful clinical course without evidence of restenosis. Follow-up coronary angiography showed some local thickening in most patients; the degree of this proliferation, however, never reached the level of significant obstruction (Figs 2 and 3). The overall local thickening was estimated to be a $10 \%$ reduction in the percentage diameter stenosis.

In recent patients, followed for an interval of less than three months (Fig. 4) and in whom no control angiography was performed, the exercise ECG was used to judge the results of the procedure. These patients also did not show any evidence of restenosis. A few patients had a thallium myocardial scintigraphy with normal flow pattern.

Among the complications encountered were local spasms immediately after the stent placement; these spasms, in general, responded to the local administration of isosorbite dinitrate or calcium antagonists. Spasm has not been a major problem since we introduced an intracoronary injection of nifedipine before the stent deployment. Important arrhythmias necessitating electrical defibrillation occurred three times during the placement. These 

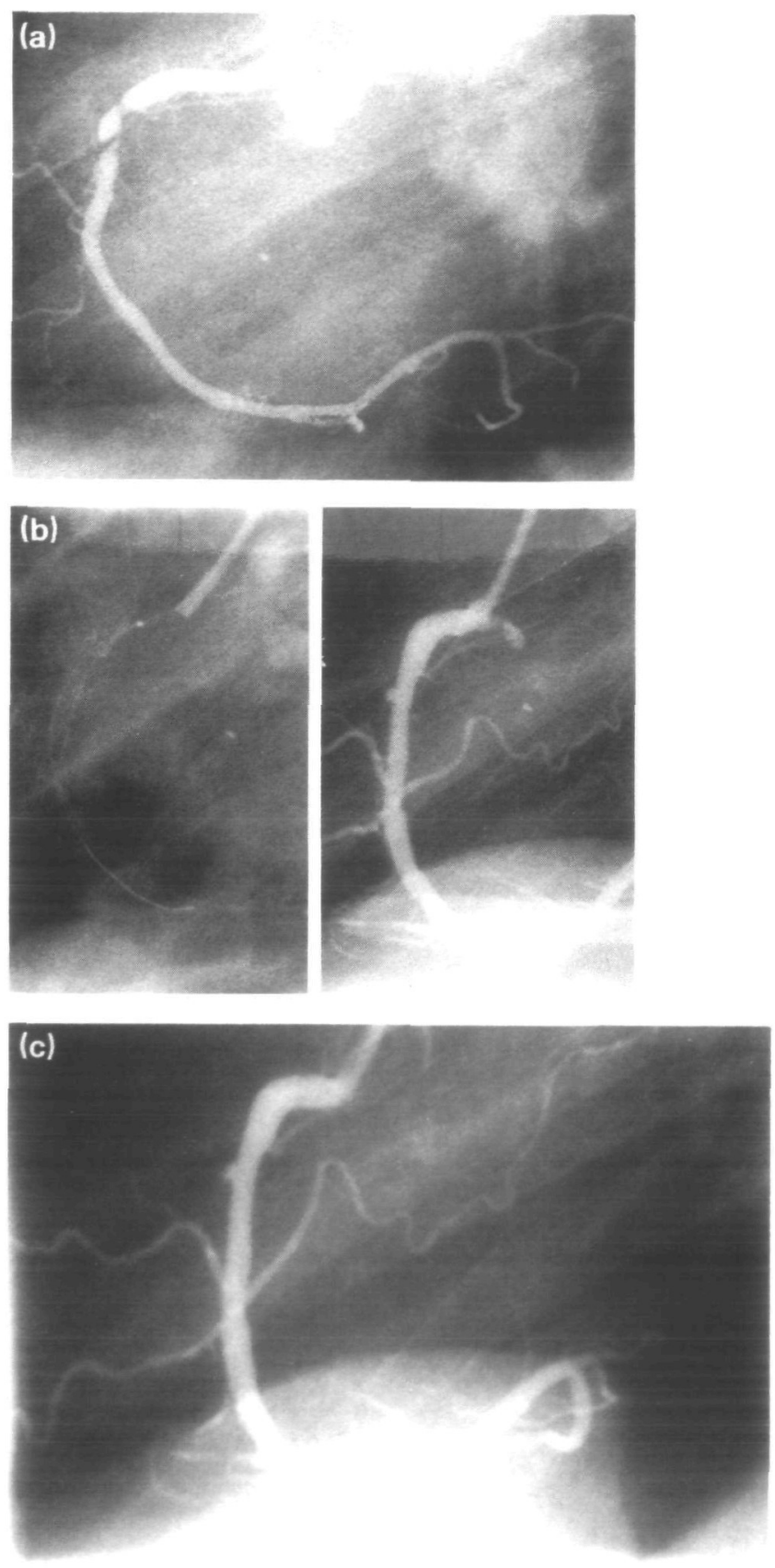

Figure 2 Restenosis of a dominant right coronary artery. (a) Three months after primary transluminal angioplasty in a 52 year old patient. (b) Immediately after angioplasty and implantation of a stent of $4 \mathrm{~mm}$ maximal diameter and $23 \mathrm{~mm}$ length; the stent is visible without contrast injection in the left part of the picture. (c) Three months after the stent implant there is no evidence of reduction of luminal diameter. 

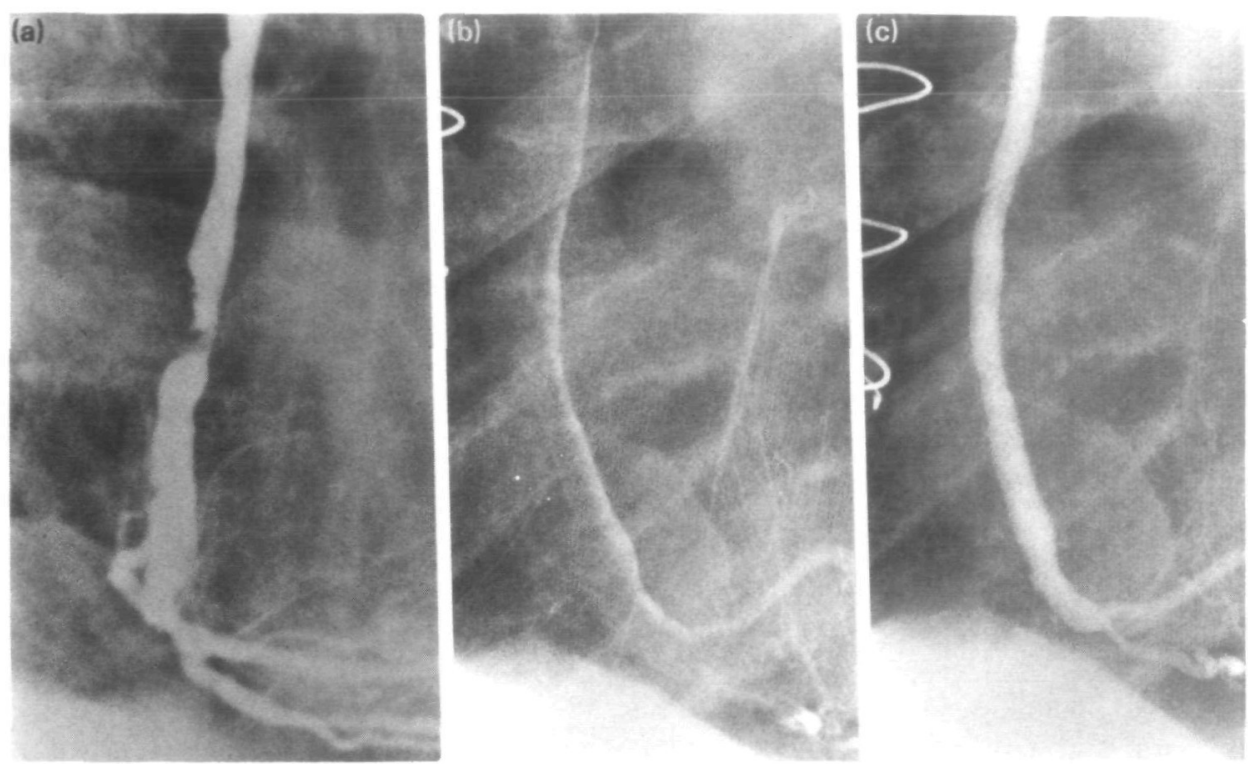

Figure 3 Complicated high grade stenosis of a bypass graft to the right coronary artery in a 51 year old patient. (a) Before stent implantation. (b) The stent of $4.5 \mathrm{~mm}$ maximal diameter and $20 \mathrm{~mm}$ length in place; opacification with contrast medium renders the stent invisible. (c) Three months after stent implantation.

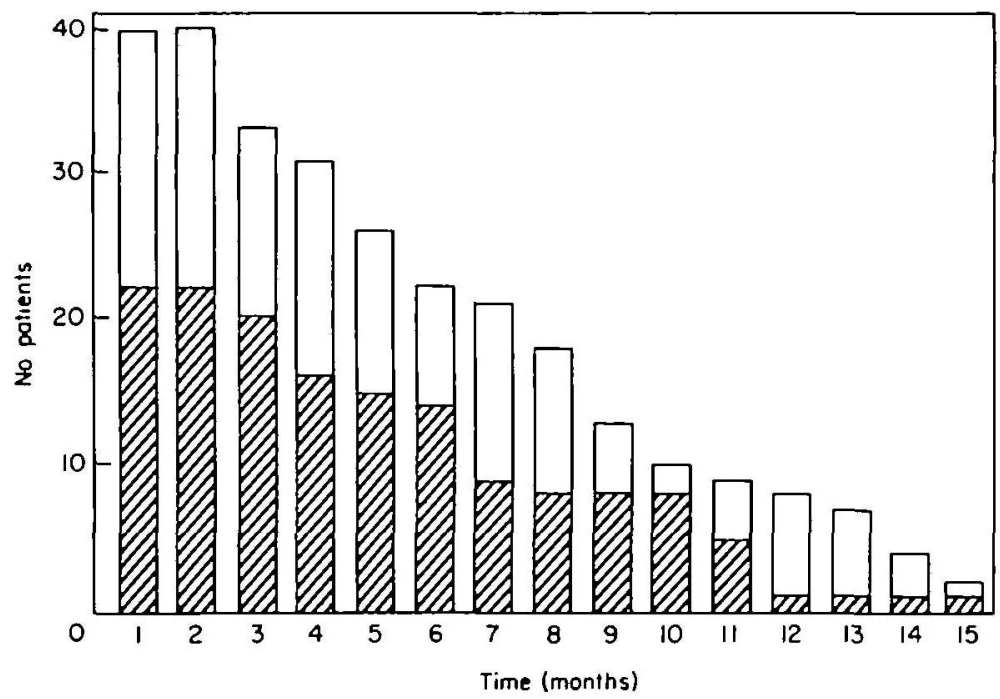

Figure 4 Histogram of the duration of the clinical and angiographic follow-up after stent implantation for restenosis. The hatched segments of the columns represent the angiographic follow-up; the open segments of the columns correspond to a clinical follow-up with ergometry and/or thallium scintigraphy.

arrhythmias are probably due to prolonged ischaemia. Among the most frequent complications was local haematoma, requiring surgical revision in two cases.

\section{Discussion}

The statistical likelihood of restenosis occurring after angioplasty, even when dealing with the second or third procedure at the same site, is quite 
high. The fact that we have not observed restenosis within a stented segment (except for one patient in whom a new stenosis was noted proximal to the stent extending into the stent) probably indicates that stenting may reduce the incidence of restenosis. The absence of a control group, however, does not permit us to draw any firm conclusion, although it would be extremely unlikely for none of the heredescribed patients not to develop restenosis after the angioplasty procedure without stenting.

Several factors may be responsible for the occurrence of restenosis: platelet adhesion or thrombus formation, localized proliferation of smooth muscle cells, and accelerated lipid uptake at the site of pressure induced intimal damage ${ }^{[5-7]}$.

Several techniques aim to improve the primary angioplasty results by smoothing the internal vascular surface or by preventing thrombosis and tissue ingrowth into the lumen after balloon dilatation or, ideally, both. The concept of improving the primary results of PTCA could be advantageous for a number of reasons: internal fissures and cracks, dissection and flaps cause blood turbulence, stasis and thrombosis, which may then be organized into restenosis. Also turbulent blood flow may accelerate the deposition of atherosclerotic material possibly through suction effects on the superficial endothelial layers. Therefore, a 'smoothing procedure' that irons out the most annoying clefts and dissections resulting from transluminal angioplasty might be the solution to a great deal of problems secondary to a highly traumatic assault on the arterial wall. The laser balloon ${ }^{[8]}$ which, according to preliminary observation, has the capacity to heat-sealing dissections and surface cracks may provide such an approach within the near future without the need for prosthetic material.

Prevention of tissue ingrowth requires either a growth barrier or suppression of the nutritional blood supply to the cellular substrate. Interstitial cell proliferation within the different layers of the arterial wall may also play a role in the genesis of stenosis; this mechanism could, theoretically, also be treated by interventions designed to impede nutritional flow via the vasa vasorum. Whether elastic recoil or spasm ${ }^{[9.10]}$ of the arterial wall plays a role in the occurrence of restenosis has never been substantiated. It seems conceivable, however, that a combination of these different mechanisms may be responsible for many cases of clinical restenosis after angioplasty. Prevention of restenosis should, therefore, deal with each of these possible modes of action.
We conclude that elastic intravascular stents may: (1) optimize the initial result of PTCA by smoothing the rugged, traumatized endothelial surface; (2) prevent elastic recoil and contraction of the arterial wall; and (3) possibly suppress nutritional flow to the cell layers that potentially form the substrate for cell proliferation. Whether the stent itself presents a significant barrier for cell proliferation seems doubtful, because the pores between the wire mesh have deliberately been chosen to be much larger than the metal surface in order to enhance rapid endothelialization. Nevertheless, the fibrous neo-endothelium may finally become a kind of physiological fence for cellular ingrowth.

Our clinical observations of up to 17 months in patients with previous documented restenosis after coronary angioplasty or deterioration of saphenous vein bypass grafts seem to corroborate these speculations. Transluminal dilatation of bypass grafts is known to yield acceptable long-term results in only half of the cases; $50 \%$ or more develop recurrence at the site of the previous lesion within the first three months. In our series of 12 bypass grafts stents, we observed no restenosis within the stented segment. The interpretation of data obtained from patients in whom the native coronary artery was stented to prevent restenosis is somewhat more complex for two reasons: first, the rate of restenosis is generally lower, and secondly we have seen one case in which coronary artery restenosis developed within three months upstream to the stent but extending into the stented segment. In addition, the overall complication rate is higher in this patient group.

Nevertheless, we are encouraged by these preliminary observations on the prevention of restenosis after coronary angioplasty. It must not be overlooked, however, that the price to pay for this achievement is still high, as reflected by the complication rate, the longer hospital stay, the higher morbidity, and the necessity of anticoagulant treatment. Improvements are necessary, not only in respect to the stent delivery technology, but also to diminish thrombogenicity and spasmogenicity of intracoronary stents.

\section{References}

[1] Meier B, King III SB, Gruentzig AR el al. Repeat coronary angioplasty. J Am Coll Cardiol 1984; 4: 463-80.

[2] Meier B. Restenosis after coronary angioplasty: review of the literature. Eur Heart J 1988; 9(Suppl C): 1-6.

[3] Sigwart U, Puel J, Mirkovitch V, Joffre F, Kappenberger L. Intravascular stents to prevent occlusion and restenosis after transluminal angioplasty. New Engl J Med 1987; 316: 701-6. 
[4] Fuster V. Personal communication, 1987.

[5] Essed CE, Van den Brand M, Becker AE. Transluminal coronary angioplasty and early restenosis - fibrocellular occlusion after wall alteration. Br Heart J 1983; 49: 393.

[6] Waller WF, Rothbaum DA, Gorfinkel HJ, Ulbright TH, Linnemeier TJ, Berger SM. Morphologic observations after percutaneous transluminal balloon angioplasty of early and late aortocoronary saphenous vein bypass grafts. J Am Coll Cardiol 1984; 4: 784.

[7] Faxon DP, Sanborn TA. Restenosis following transluminal angioplasty in expenmental atherosclerosis. Artenosclerosis 1984; 4 : 189.
[8] Jenkins RD, Sinclair IN, Anand R, Spears JR. Laser balloon angioplasty: thermal profile for in vitro welding of neointimal arterial separations. J Am Coll Cardiol $1987 ; 9(2): 105 \mathrm{~A}$.

[9] Hollman J, Austin GE, Gruentzig AR, Douglas JS Jr, King SB III. Coronary artery spasm at the site of angioplasty in the first 2 months after successful percutaneous transluminal coronary angioplasty. J Am Coll Cardiol 1983; 2: 1039-45.

[10] Probst P, Zangl W, Baumgartner C, Pachinger O. The inducibility of coronary artery spasm after successful PTCA. Eur Heart J 1988; 9(Suppl C): 19-20. 